\title{
Performance of a Class of Bayesian Shrinkage Estimators Based on Rayleigh Record Data under Reflected Gamma Loss Function
}

\author{
Mehran Naghizadeh Qomi ${ }^{1}$, Sanku Dey ${ }^{2}$, and Monir Fathollahi ${ }^{1}$ \\ ${ }^{1}$ Department of Statistics, University of Mazandaran, Babolsar, Iran. \\ ${ }^{2}$ Department of Statistics, St. Anthony's College, Shillong, Meghalaya, India.
}

Received: 24/07/2018, Revision received: 28/03/2019, Published online: 29/08/2019

\begin{abstract}
This article addresses the problem of Bayesian shrinkage estimation for the Rayleigh scale parameter based on record values under the reflected gamma loss (RGL) function. A class of Bayesian shrinkage estimators using prior point information is constructed. The risk functions of the maximum likelihood estimator (MLE) and proposed Bayesian shrinkage estimator are derived under the RGL function. The performance of Bayesian shrinkage estimator is compared with the MLE numerically and graphically. One data set has been analyzed to illustrate the performance of the Bayesian shrinkage estimator.
\end{abstract}

Keywords. Bayesian Shrinkage Estimator, Rayleigh Distribution, Records, Reflected Gamma Loss Function.

MSC: 62F10; 62F15.

Corresponding Author: Mehran Naghizadeh Qomi (m.naghizadeh@umz.ac.ir)

Sanku Dey (sanku_dey2k2003@yahoo.co.in) 


\section{Introduction}

Consider a sequence of independent and identically distributed (i.i.d.) random variables as $\left\{X_{i}, i \geq 1\right\}$ having a cumulative distribution function (CDF) $F$ and a probability density function (PDF) $f$. An observation $X_{j}$ is an upper record value if $X_{j}>X_{i}$ for every $i<j$. An analogous definition deals with lower record values. Examples of application areas from data of this type include industrial stress testing, meteorological analysis, sporting and athletic events, and oil and mining surveys; see Arnold et al. (1998). We denote the $m$ th upper record value by $R_{m}$. The joint density of the first $m$-records $\mathbf{R}=\left(R_{1}, \ldots, R_{m}\right)$ is given by

$$
f_{R_{1}, \cdots, R_{m}}\left(r_{1}, \ldots, r_{m}\right)=f\left(r_{m}\right) \prod_{i=1}^{m-1} \frac{f\left(r_{i}\right)}{1-F\left(r_{i}\right)}, \quad r_{1}<r_{2}<\cdots<r_{m}
$$

Also, the marginal p.d.f. of the $m$ th record, $R_{m}$, is given by

$$
f_{R_{m}}(x)=\frac{[-\log (1-F(x))]^{m-1}}{(m-1) !} f(x) .
$$

Rayleigh distribution has wide applicability in the field of acoustics, communication engineering, in the life-testing of electro-vacuum devices, in reliability theory and survival analysis and so on. Since the introduction of Rayleigh distribution in 1880 , researchers have shown immense interest in this distribution because of its many uses in different areas of science and technology. The most important feature of the Rayleigh distribution is that its failure rate is an increasing function of time, i.e., if the failure times are distributed according to the Rayleigh law, intense aging of the equipment/item takes place. Detailed work on Rayleigh distribution can be found in the works of authors such as Dey and Das (2007), Dey (2009), Dey and Day (2014), Dey et al. (2017) and the references cited therein have carried out extensive studies as related to the estimation, prediction and several other inferences with respect to Rayleigh distribution.

Let $X$ have a one parameter Rayleigh distribution with PDF

$$
f(x \mid \theta)=\frac{x}{\theta} e^{-\frac{x^{2}}{2 \theta}}, \quad x>0, \theta>0,
$$


and the corresponding $\mathrm{CDF}$ as

$$
F(x \mid \theta)=1-e^{-\frac{x^{2}}{2 \theta}}, \quad x>0 .
$$

If $\mathbf{R}=\left(R_{1}, \ldots, R_{m}\right)$ be the first $m$-records samples from the Rayleigh distribution, then from (1.1) and (1.2), the likelihood function of $\theta$ based on $\mathbf{R}=\left(R_{1}, \ldots, R_{m}\right)$ at $\mathbf{r}=\left(r_{1}, \ldots, r_{m}\right)$ is given by

$$
L(\theta \mid \mathbf{r})=\left(\prod_{i=1}^{m} \frac{r_{i}}{\theta}\right) e^{-\frac{r_{m}^{2}}{2 \theta}}, \quad \theta>0 .
$$

Then, the MLE of $\theta$ can be derived from the equation $\frac{\partial L(\theta \mid \mathbf{r})}{\partial \theta}=0$, which is given by $\hat{\theta}=R_{m}^{2} /(2 m)$.

In many situations, the researcher has some prior information about the parameter of interest $\theta$ in the form of a point guess value $\theta_{0}$ and sample information that provides the estimator, MLE, $\hat{\theta}$. However, the researcher can combine non-sample information $\left(\theta_{0}\right)$ and sample information $(\hat{\theta})$ and construct a new estimator with a hope that it will perform better than the MLE. Thompson (1968) considered the linear point shrinkage estimator as

$$
\hat{\theta}_{s}=k\left(\hat{\theta}-\theta_{0}\right)+\theta_{0}=k \hat{\theta}+(1-k) \theta_{0}, \quad k \in[0,1],
$$

where $k$ is known as a shrinkage factor. The value of $k$ near to zero (one) implies a strong belief in the guess value $\theta_{0}$ (sample values). Significant attention has been paid to the problem of shrinkage estimation, see Prakash and Singh (2008), Naghizadeh Qomi and Barmoodeh (2015), Belaghi et al. (2014), Belaghi et al. (2015 a, b), Kiapour and Naghizadeh (2016), Baklizi et al. (2016), Naghizadeh Qomi (2017a), Safarian et al. (2018) and Volterma et al. (2018).

In the Bayesian approach of estimation, a Bayesian estimator is derived by employing a flexible prior distribution for the parameter of interest. Prakash and Singh (2009) considered the problem of Bayesian shrinkage estimation in Weibull type-II censored data under the squared error loss (SEL) and linear-exponential (LINEX) loss functions in the presence of a point guess value of true parameter. Dey et al. (2015) dealt with the Bayesian shrinkage estimation for the progressively censored samples from Rayleigh distribution under a general entropy loss function. Naghizadeh Qomi (2017b) used 


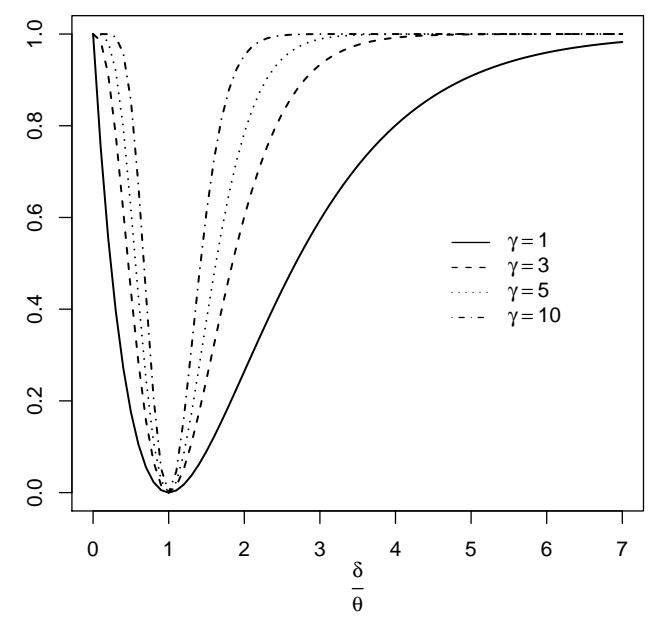

Figure 1: The plot of the RGL function for $l=1$ and selected values of $\gamma=1,3,5,10$.

a squared log error loss (SLEL) function for obtaining the Bayesian shrinkage estimators for the scale-parameter of a Rayleigh distribution based on type-II censored data. Kiapour (2017) considered the Bayesian shrinkage estimation for exponential distribution under a precautionary loss function. The loss functions proposed in the aforementioned articles are not bounded. In some estimation problems, the use of an unbounded loss function may be inappropriate. For example, in estimating the mean life $\theta$ of the components of an aircraft, the amount of loss for estimating the parameter $\theta$ by an estimator is essentially bounded. In this paper, we consider the reflected gamma loss (RGL) function for estimating the scale parameter $\theta$ as

$$
L(\theta, \delta)=l\left[1-\left(\frac{\delta}{\theta}\right)^{\gamma} e^{-\gamma\left(\frac{\delta}{\theta}-1\right)}\right]
$$

where $l>0$ is the maximum loss, $\gamma>0$ is a shape parameter and $\delta$ is an estimator of $\theta$. Towhidi and Behboodian $(1999,2002)$, Meghnatisi and Nematollahi (2009) and Naghizadeh Qomi et al. (2015) considered this loss function in some estimation problems. It is noticed that the RGL function is constructed by flipping a gamma density upside down, see Figure 1. We observe that the RGL function: 
1. Is bounded by 0 and $l$.

2. Is an asymmetric function of $\delta / \theta$ but not convex in $\delta / \theta$ and has a unique minimum 0 for $\delta / \theta=1$, is strictly decreasing on $(0,1)$ and increasing on $(1, \infty) . L(0)=l$ and $\lim _{\frac{\delta}{\theta} \rightarrow \infty} L\left(\frac{\delta}{\theta}\right)=l$.

3. Is downside damaging, because $L(\delta-\varepsilon) \geq L(\delta+\varepsilon), \forall \varepsilon>0$, and then underestimation is penalized more heavily than over-estimation.

The uniqueness of this study comes from the fact that thus far, no attempt has been made to compare the Bayesian estimator and the generalized Bayesian shrinkage estimator using a RGL function with MLE based on record values. Further, empirical Bayesian shrinkage estimator has been obtained.

The rest of the paper is organized as follows. In Section 2, we obtain the Bayesian estimator of $\theta$ under RGL function. In Section 3, a generalized Bayesian shrinkage estimator is constructed under the RGL function. A comparative study is performed between the proposed Bayesian shrinkage estimator and the MLE in Section 4. In Section 5, a data set has been analyzed to illustrate the performance of the Bayesian shrinkage estimator. Finally, in Section 6, we end the paper with some remarks.

\section{The Bayesian Estimator of $\theta$ under RGL}

Let $\mathbf{R}=\left(R_{1}, \cdots, R_{m}\right)$ be the first $m$-record data arising from a sequence of i.i.d. Rayleigh variable with PDF given in (1.2). Consider the natural conjugate IGamma $(a, b)$-prior for $\theta$ with PDF

$$
\pi^{a, b}(\theta)=\frac{a^{b} e^{-\frac{a}{2 \theta}}}{2^{b} \Gamma(b) \theta^{b+1}}, \quad \theta>0, a>0, b>0
$$

where $\Gamma(b)=\int_{0}^{\infty} t^{b-1} e^{-t} d t$ is the gamma function. When no prior information about the parameter $\theta$ is available, we may use the noninformative Jeffreys prior $\pi^{0,0}(\theta)=$ $1 / \theta, \theta>0$. Combining the likelihood function (1.3) with the prior (2.1), the posterior PDF of $\theta$ given $\mathbf{R}$ is $\operatorname{IGamma}\left(R_{m}^{2}+a, m+b\right)$ of the form

$$
\pi(\theta \mid \mathbf{R})=\frac{\left(R_{m}^{2}+a\right)^{m+b} e^{-\left(R_{m}^{2}+a\right) / 2 \theta}}{\Gamma(m+b) 2^{m+b} \theta^{m+b+1}} .
$$


The posterior risk of $\delta=\delta(\mathbf{R})$ under the RGL is given by

$$
\begin{aligned}
\rho(\delta) & =E[L(\theta, \delta) \mid \mathbf{R}]=1-E\left[\left(\frac{\delta}{\theta}\right)^{\gamma} e^{-\gamma\left(\frac{\delta}{\theta}-1\right)} \mid \mathbf{R}\right] \\
& =1-\int_{0}^{\infty}\left(\frac{\delta}{\theta}\right)^{\gamma} e^{-\gamma\left(\frac{\delta}{\theta}-1\right)} \frac{\left(R_{m}^{2}+a\right)^{m+b} e^{-\left(R_{m}^{2}+a\right) / 2 \theta}}{\Gamma(m+b) 2^{m+b} \theta^{m+b+1}} d \theta \\
& =1-\frac{A \delta \gamma}{\left(R_{m}^{2}+a+2 \gamma \delta\right)^{m+b+\gamma}},
\end{aligned}
$$

where $A=(2 e)^{\gamma}\left(R_{m}^{2}+a\right)^{m+b} \Gamma(m+b+\gamma) / \Gamma(m+b)$. Note that we take $l=1$, without loss of generality. The Bayesian estimator $\hat{\theta}_{B}(\mathbf{R})$ is obtained by minimizing the posterior risk with respect to $\delta$. Doing some calculations, we obtain the Bayesian estimator as

$$
\hat{\theta}_{B}(\mathbf{R})=\frac{R_{m}^{2}+a}{2(m+b)}=\frac{2 m \hat{\theta}+a}{2(m+b)}
$$

which does not depends on $\gamma$. By choosing $a=b=0$, the conjugate inverse Gamma prior for $\theta$ converts to a Jeffreys prior as a limiting prior and then the generalized Bayesian estimator of $\theta$ becomes $\hat{\theta}$, the MLE of $\theta$.

\section{A Class of Bayesian Shrinkage Estimators}

Suppose that we have some a priori information about the parameter $\theta$ as a point guess $\theta_{0}$. Following Prakash and Singh (2009) we select the parameters $a$ and $b$ of the prior distribution (2.1) such that $E\left[\hat{\theta}_{B}(\mathbf{R})\right]=\theta_{0}$ or equivalently

$$
\frac{2 m E[\hat{\theta}]+a}{2(m+b)}=\frac{2 m \theta_{0}+a}{2(m+b)}=\theta_{0}
$$

which implies that $a=2 b \theta_{0}$. By substituting this value of $a$ in $\hat{\theta}_{B}(\mathbf{R})$, we obtain an estimator of the form

$$
\hat{\theta}_{B S}(\mathbf{R})=\lambda \hat{\theta}+(1-\lambda) \theta_{0}
$$


where $\lambda=m /(m+b)$. The estimator $\hat{\theta}_{B S}(\mathbf{R})$ is similar to the shrinkage estimator in (1.4) and we consider it as a Bayesian shrinkage estimator of $\theta$. Note that if $b \rightarrow 0$, then $\hat{\theta}_{B S}(\mathbf{R}) \rightarrow \hat{\theta}$, whilst for $b \rightarrow \infty$, we have $\hat{\theta}_{B S}(\mathbf{R}) \rightarrow \theta_{0}$.

Now, consider a class of Bayesian shrinkage estimators of the form

$$
\hat{\theta}_{G B S}^{(k)}(\mathbf{R})=\frac{m+b k}{m+b} \hat{\theta}+\frac{b(1-k)}{m+b} \theta_{0}=\lambda^{\star} \hat{\theta}+\left(1-\lambda^{\star}\right) \theta_{0}
$$

where $\lambda^{\star}=(m+b k) /(m+b)$ and $k$ lies in $[0,1]$. We called the estimator $\hat{\theta}_{G B S}^{(k)}(\mathbf{R})$ as generalized Bayesian shrinkage estimator. Note that for $k=0$, we have $\hat{\theta}_{G B S}^{(0)}(\mathbf{R})=$ $\hat{\theta}_{B S}(\mathbf{R})$.

The risk function of $\hat{\theta}_{G B S}^{(k)}(\mathbf{R})$ under the RGL function is

$$
\begin{aligned}
R\left(\theta, \hat{\theta}_{G B S}^{(k)}\right) & =1-E\left[\left(\frac{\hat{\theta}_{G B S}^{(k)}}{\theta}\right)^{\gamma} e^{-\gamma\left(\frac{\hat{\theta}_{G B S}^{(k)}}{\theta}-1\right)}\right] \\
& =1-e^{\gamma} E\left[\left(\frac{\lambda^{\star} \hat{\theta}+\left(1-\lambda^{\star}\right) \theta_{0}}{\theta}\right)^{\gamma} e^{-\gamma\left(\frac{\lambda \hat{\theta}+(1-\lambda) \theta_{0}}{\theta}\right)}\right] \\
& =1-e^{\gamma} E\left[\left(\frac{\lambda^{\star} U}{2 m}+\left(1-\lambda^{\star}\right) \Delta\right)^{\gamma} e^{-\gamma\left(\frac{\lambda^{\star} U}{2 m}+\left(1-\lambda^{\star}\right) \Delta\right)}\right] \\
& =1-e^{\gamma\left[1-\left(1-\lambda^{\star}\right) \Delta\right]} \int_{0}^{\infty}\left(\frac{\lambda^{\star} u}{2 m}+\left(1-\lambda^{\star}\right) \Delta\right)^{\gamma} e^{\frac{-\gamma \lambda^{\star} u}{2 m}} g(u) d u,
\end{aligned}
$$

where $\Delta=\theta_{0} / \theta$ and $g(u)$ is the density of $U=2 m \hat{\theta} / \theta \sim \chi_{2 m}^{2}$.

The risk function of $\hat{\theta}$ under the RGL function is

$$
\begin{aligned}
R(\theta, \hat{\theta}) & =1-E\left[\left(\frac{\hat{\theta}}{\theta}\right)^{\gamma} e^{-\gamma\left(\frac{\hat{\theta}}{\theta}-1\right)}\right] \\
& =1-\left(\frac{e}{2 m}\right)^{\gamma} E\left[U^{\gamma} e^{-\frac{\gamma u}{2 m}}\right]=1-\left(\frac{e}{2 m}\right)^{\gamma} \int_{0}^{\infty} u^{\gamma} e^{-\frac{\gamma u}{2 m}} g(u) d u \\
& =1-\frac{e^{\gamma} m^{m} \Gamma(m+\gamma)}{(m+\gamma)^{m+\gamma} \Gamma(m)} .
\end{aligned}
$$




\section{A Comparative Study}

In this section, we evaluate the performance of the proposed Bayesian shrinkage estimator and ML estimator. For comparison purposes, the relative efficiency (R.E.) of $\hat{\theta}_{G B S}^{(k)}$ with respect to $\hat{\theta}$ has been calculated as

$$
R E\left(\hat{\theta}_{G B S^{\prime}}^{(k)} \hat{\theta}\right)=\frac{R(\theta, \hat{\theta})}{R\left(\theta, \hat{\theta}_{G B S}^{(k)}\right)} .
$$

Figure 2 shows the shape of RE for selected values of $k=0,0.3,0.6,0.9, m=2(1) 5$, $\gamma=1$ and $b=3$ with respect to $\Delta$. Note that we used the notation low(step)up for the presentation of values. It is observed that the generalized Bayesian shrinkage estimator performs well with compared to the MLE for $\Delta$ closer 1 . Moreover, the generalized Bayesian shrinkage estimator with $k=0$, i.e., $\hat{\theta}_{B S}$ is better than other estimators in the neighborhood of $\theta_{0}$.

The range of $\Delta$ that of $\hat{\theta}_{B S}$ dominates $\hat{\theta}$ (the so-called effective interval) and the values of $\Delta_{\text {max }}$ for which RE is maximum are summarized in Table 1 . It is observed that the length of effective intervals for fixed $m$ and $\gamma$ decreases when $b$ increases. Also, when the values of $m$ and $b$ are held fixed, the length decreases when $\gamma$ increases.

We also plotted the R.E. of $\hat{\theta}_{B S}$ and $\hat{\theta}$ in Figures 3-4 for various values of $m, \gamma, b$ and $\Delta$. From these figures, the following conclusions can be drawn:

1. The Bayesian shrinkage estimator performs well with respect to the MLE for $\Delta$ closer to 1 .

2. For fixed $m$ and $\gamma$, the Bayesian shrinkage estimators with a larger value of $b$ outperforms other estimators in the neighborhood of $\theta_{0}$.

3. The R.E. is decrease with respect to $m$ when $b$ and $\gamma$ are held fixed and $\Delta$ is close to one.

We also plot the R.E. in Figure 5 for selected values of $m$ and $b$ with respect to $\gamma$ when $\Delta=1$. It is observed from Figure 5 that the R.E. is decreasing with respect to $\gamma$ for fixed values of $m$ and $b$. 


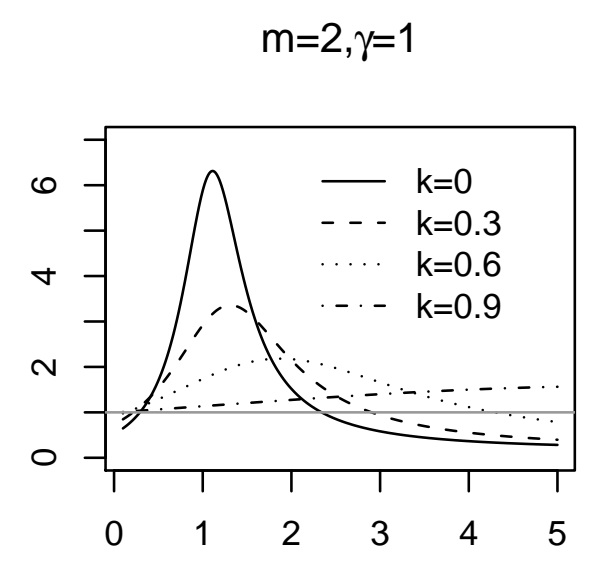

$\Delta$

$$
\mathrm{m}=4, \gamma=1
$$

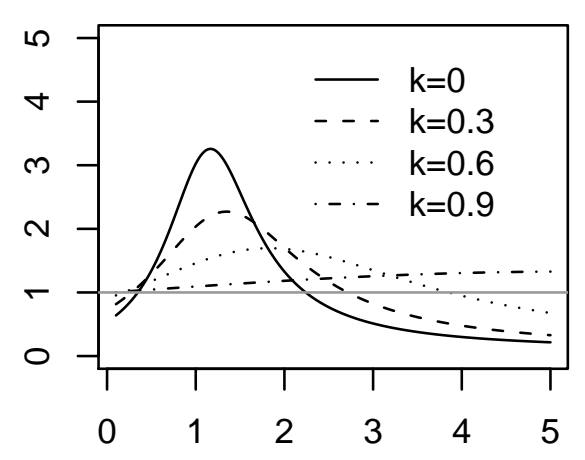

$\Delta$ $m=3, \gamma=1$

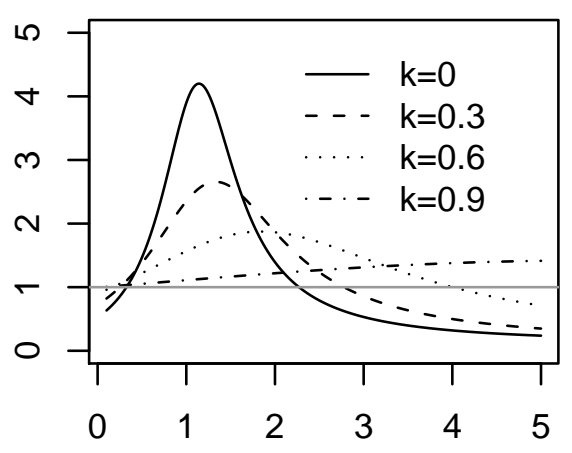

$\Delta$

$$
m=5, \gamma=1
$$

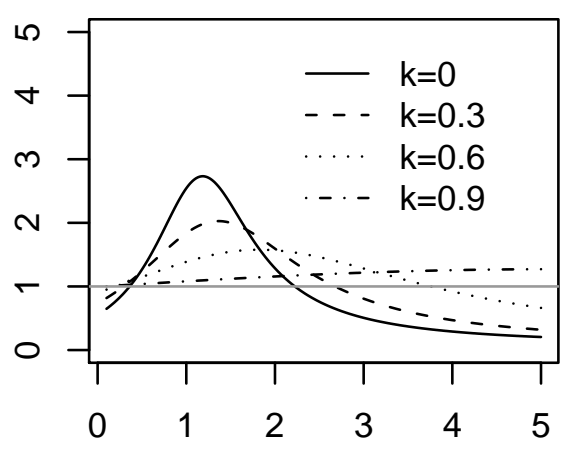

$\Delta$

Figure 2: Plots of R.E. between $\hat{\theta}_{G B S}^{(k)}$ and $\hat{\theta}$ for selected values of $k=0,0.3,0.6,0.9, m=2(1) 5$, $b=3$ and $\gamma=1$ with respect to $\Delta$. 


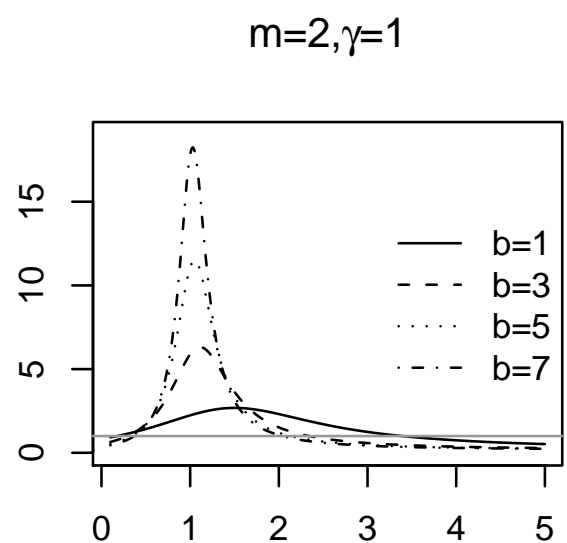

$\Delta$

$$
\mathrm{m}=4, \gamma=1
$$

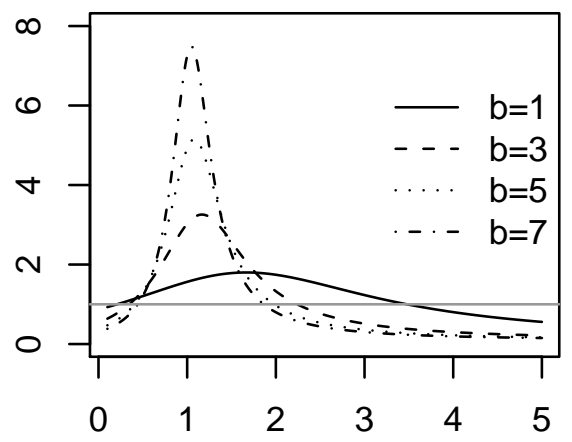

$\Delta$ $m=3, \gamma=1$

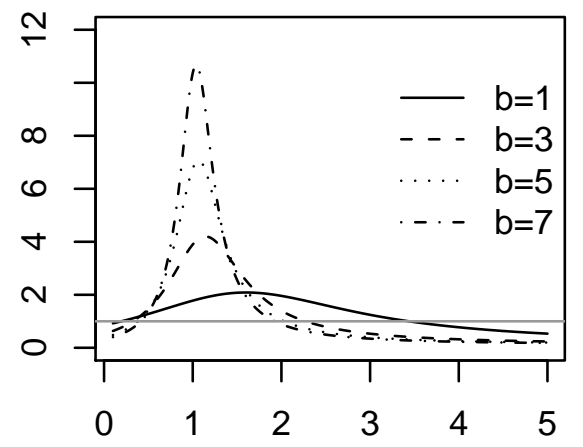

$\Delta$

$$
m=5, \gamma=1
$$

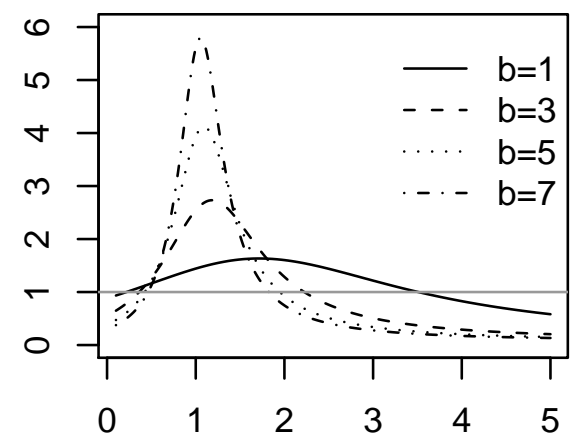

$\Delta$

Figure 3: Plots of R.E. between $\hat{\theta}_{B S}$ and $\hat{\theta}$ for selected values of $m=2(1) 5, b=1,3,5,7$ and $\gamma=1$ with respect to $\Delta$. 


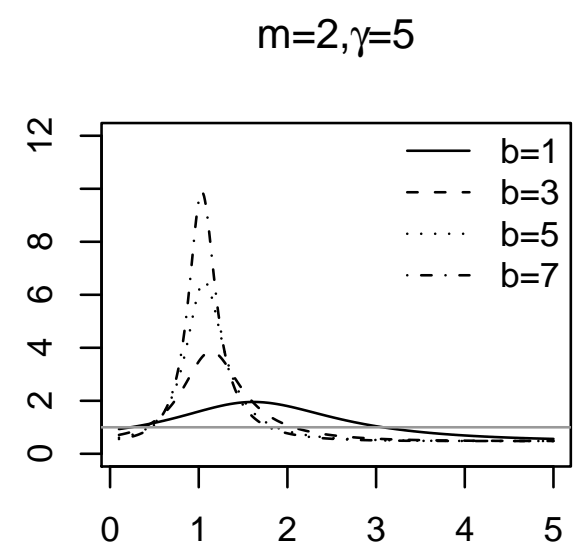

$\Delta$

$$
\mathrm{m}=4, \gamma=5
$$

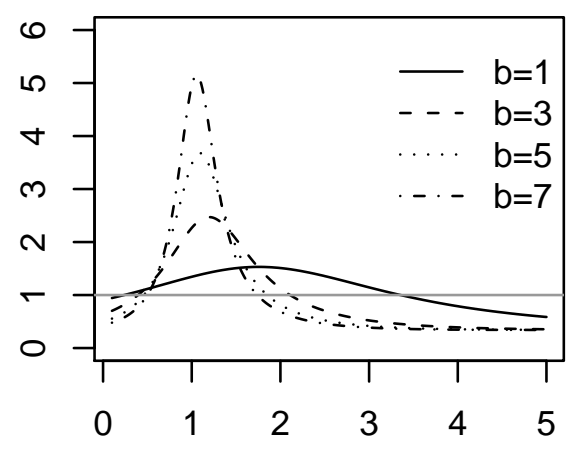

$\Delta$ $m=3, \gamma=5$

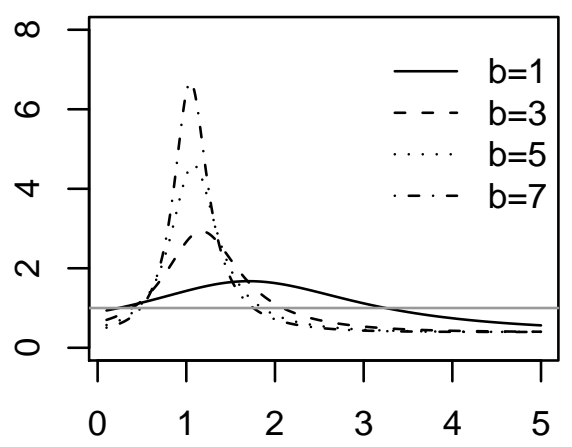

$\Delta$

$$
m=5, \gamma=5
$$

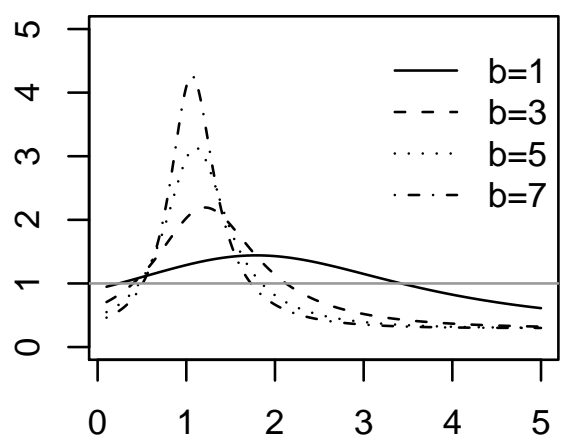

$\Delta$

Figure 4: Plots of R.E. between $\hat{\theta}_{B S}$ and $\hat{\theta}$ for selected values of $m=2(1) 5, b=1,3,5,7$ and $\gamma=5$ with respect to $\Delta$. 


$$
\mathrm{m}=2
$$

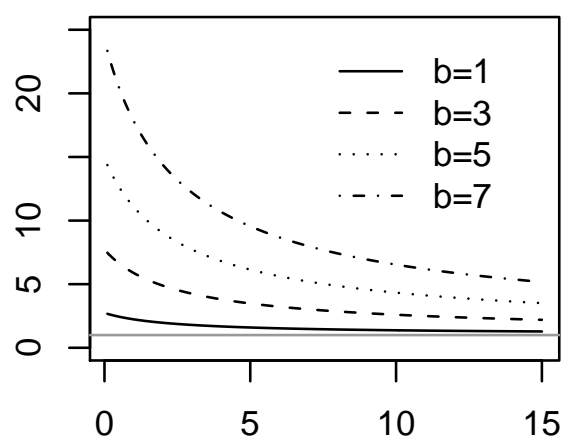

$\gamma$

$\mathrm{m}=4$

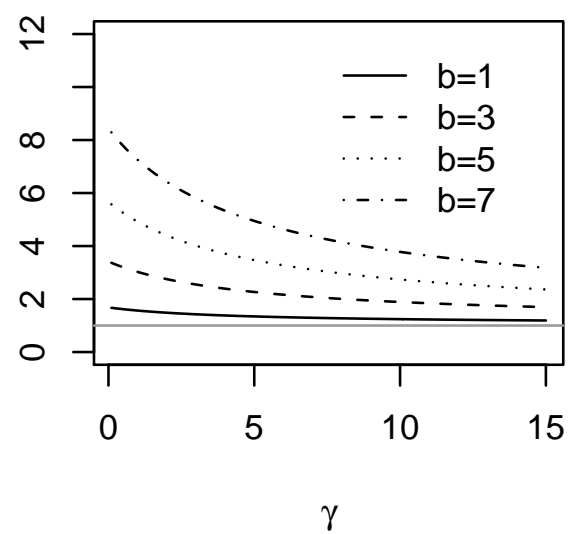

$m=3$

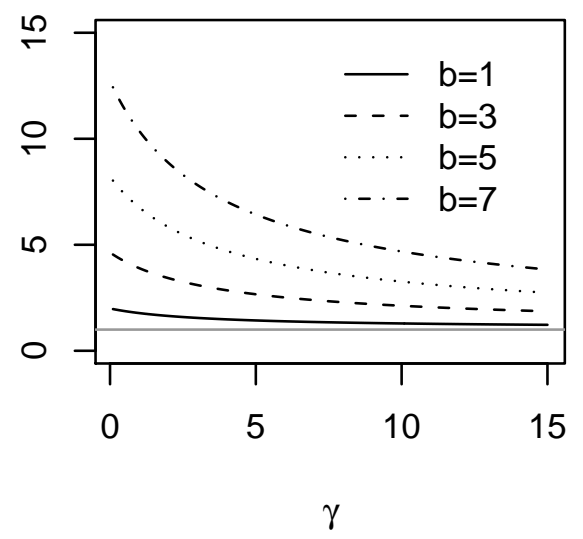

$m=5$

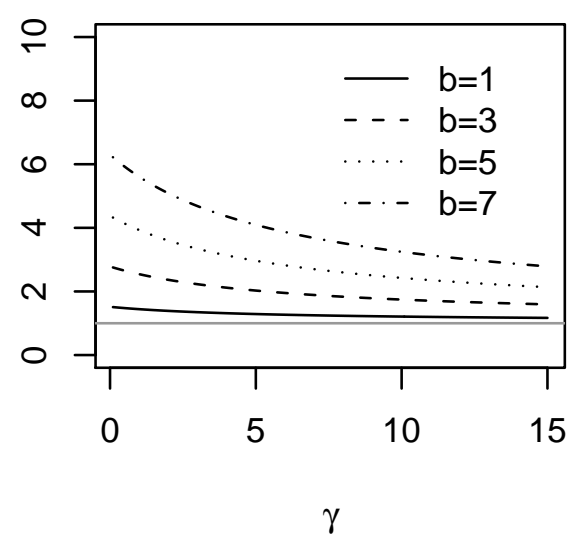

Figure 5: Plots of R.E. between $\hat{\theta}_{B S}$ and $\hat{\theta}$ for selected values of $m=2(1) 5 b=1,3,5,7$ and $\Delta=1$ with respect to $\gamma$. 
Table 1: The value of $\Delta_{\max }$ and the range of $\Delta$ that $\hat{\theta}_{B S}$ dominates $\hat{\theta}$.

\begin{tabular}{|c|c|c|c|c|c|c|c|c|c|}
\hline \multirow[b]{2}{*}{$m$} & & \multicolumn{2}{|l|}{$\gamma=1$} & \multicolumn{2}{|l|}{$\gamma=3$} & \multicolumn{2}{|l|}{$\gamma=5$} & \multicolumn{2}{|l|}{$\gamma=7$} \\
\hline & $b$ & $\Delta_{\max }$ & {$\left[\Delta_{1}, \Delta_{2}\right]$} & $\Delta_{\max }$ & {$\left[\Delta_{1}, \Delta_{2}\right]$} & $\Delta_{\max }$ & {$\left[\Delta_{1}, \Delta_{2}\right]$} & $\Delta_{\max }$ & {$\left[\Delta_{1}, \Delta_{2}\right]$} \\
\hline \multirow[t]{4}{*}{2} & 1 & 1.500 & {$[0.1753,3.3869]$} & 1.5674 & {$[0.2135,3.1809]$} & 1.6159 & {$[0.2332,3.0765]$} & 1.6533 & {$[0.2450,3.0125]$} \\
\hline & 3 & 1.1111 & {$[0.2914,2.3404]$} & 1.1268 & {$[0.3464,2.1635]$} & 1.1395 & {$[0.3792,2.0663]$} & 1.1500 & {$[0.4012,2.0029]$} \\
\hline & 5 & 1.0500 & {$[0.3414,2.1290]$} & 1.0566 & {$[0.4000,1.9643]$} & 1.0623 & {$[0.4363,1.8714]$} & 1.0673 & {$[0.4618,1.8095]$} \\
\hline & 7 & 1.0280 & {$[0.3700,2.0381]$} & 1.0319 & {$[0.4297,1.8806]$} & 1.0350 & {$[0.4674,1.7905]$} & 1.0378 & [0.4941,1.7299] \\
\hline \multirow[t]{4}{*}{3} & 1 & 1.600 & {$[0.1961,3.4366]$} & 1.6606 & {$[0.2214,3.3150]$} & 1.7049 & {$[0.2355,3.2489]$} & 1.7389 & {$[0.2445,3.2076]$} \\
\hline & 3 & 1.1428 & {$[0.3251,2.2713]$} & 1.1590 & {$[0.3637,2.1573]$} & 1.1722 & {$[0.3881,2.0887]$} & 1.1833 & {$[0.4051,2.0421]$} \\
\hline & 5 & 1.0668 & {$[0.3816,2.0297]$} & 1.0739 & {$[0.4235,1.9222]$} & 1.0803 & {$[0.4511,1.8555]$} & 1.0859 & {$[0.4711,1.8089]$} \\
\hline & 7 & 1.0385 & {$[0.4145,1.9244]$} & 1.0429 & {$[0.4575,1.8216]$} & 1.0465 & {$[0.4864,1.7567]$} & 1.0498 & {$[0.5067,1.7108]$} \\
\hline \multirow[t]{4}{*}{4} & 1 & 1.6668 & {$[0.2092,3.4776]$} & 1.7180 & {$[0.2272,3.3993]$} & 1.7558 & {$[0.2380,3.3545]$,} & 1.7849 & {$[0.2452,3.3259]$} \\
\hline & 3 & 1.1666 & {$[0.3464,2.2402]$} & 1.1810 & {$[0.3753,2.1599]$} & 1.1945 & {$[0.3944,2.1086]$} & 1.2051 & {$[0.4080,2.0726]$} \\
\hline & 5 & 1.0800 & {$[0.4071,1.9785]$} & 1.0874 & {$[0.4389,1.9015]$} & 1.0938 & {$[0.4609,1.8505]$} & 1.099 & {$[0.4771,1.8137]$} \\
\hline & 7 & 1.0476 & {$[0.4429,1.8634]$} & 1.051 & {$[0.4757,1.7892]$} & 1.0556 & {$[0.4989,1.7393]$} & 1.059 & {$[0.5164,1.7027]$} \\
\hline \multirow[t]{4}{*}{5} & 1 & 1.7142 & {$[0.2182,3.5097]$} & 1.7570 & {$[0.2317,3.4560]$} & 1.7892 & {$[0.2403,3.4239]$} & 1.8139 & {$[0.2462,3.4029]$} \\
\hline & 3 & 1.1851 & {$[0.3614,2.2239]$} & 1.199 & {$[0.3838,2.1643]$} & 1.2107 & {$[0.3992,2.1244]$} & 1.2204 & {$[0.4105,2.0957]$} \\
\hline & 5 & 1.0908 & {$[0.4250,1.9482]$} & 1.098 & {$[0.4501,1.8897]$} & 1.1042 & {$[0.4681,1.8493]$} & 1.1097 & {$[0.4817,1.8194]$} \\
\hline & 7 & 1.0549 & {$[0.4628,1.8257]$} & 1.059 & {$[0.4889,1.7690]$} & 1.0630 & {$[0.5080,1.7291]$} & 1.0664 & {$[0.5228,1.6990]$} \\
\hline
\end{tabular}

\section{An illustrative Example}

The data set consists of 16 observations. The data describes the survival times (in days) of a group of lung cancer patients (see Lawless (2003)). The data are as follows

$6.96,9.30,6.96,7.24,9.30,4.90,8.42,6.05,10.18$, $6.82,8.58,7.77,11.94,11.25,12.94,12.94$.

We estimate the unknown parameter of the model by maximum likelihood and the value is $\hat{\theta}=41.9355$. For checking the validity of the Rayleigh distribution, we have used the Kolmogorov-Smirnov (K-S) statistic. It is observed that the K-S distance is $\mathrm{K}-\mathrm{S}=0.3007$ with a corresponding $\mathrm{p}$-value $=0.1108$. From this original data set, we have extracted five upper records for our data analysis. The record data are

\section{$6.96,9.30,10.18,11.94,12.94$.}

Therefore, we have $r_{5}=12.94$, which indicates that the MLE of $\theta$ is $\hat{\theta}=\frac{r_{5}^{2}}{10}=16.7443$. We consider MLE as the true value of $\theta$ and summarize the Bayesian shrinkage estimates and their risks for selected underestimated and overestimated values of $\theta_{0}=10(10) 50, b=0,1,5(5), 20$ and $\gamma=1,5$ in Table 2 . We observe from Table 2 that the Bayesian shrinkage estimates increase when the value of $b$ increases. Also, the risk of the Bayesian shrinkage estimators decreases when $\theta_{0}=20$. Moreover, the Bayesian 
Table 2: The Bayesian shrinkage estimates and their risks (in parenthesis) for selected values of $\theta_{0}$ and $b$.

\begin{tabular}{|c|c|c|c|c|c|c|c|}
\hline \multirow[b]{2}{*}{$\theta_{0}$} & \multirow[b]{2}{*}{$\gamma$} & \multicolumn{6}{|l|}{$b$} \\
\hline & & 0 & 1 & 5 & 10 & 15 & 20 \\
\hline \multirow[t]{2}{*}{10} & 1 & $16.7443(0.0896)$ & $15.6202(0.0734)$ & $13.3721(0.0569)$ & $12.2480(0.0605)$ & $11.6860(0.0664)$ & $11.3480(0.0715)$ \\
\hline & 5 & $16.7443(0.2987)$ & $15.6202(0.2624)$ & $13.3721(0.2268)$ & $12.2480(0.2495)$ & $11.6860(0.2771)$ & $11.3480(0.2995)$ \\
\hline \multirow[t]{2}{*}{20} & 1 & $16.7443(0.0896)$ & $17.2869(0.0586)$ & $18.3721(0.0230)$ & $18.9147(0.0155)$ & $19.1860(0.0139)$ & $19.3488(0.0136)$ \\
\hline & 5 & $16.7443(0.2987)$ & $17.2869(0.2211)$ & $18.3721(0.0992)$ & $18.9147(0.0705)$ & $19.1860(0.0651)$ & $19.3488(0.0645)$ \\
\hline \multirow[t]{2}{*}{30} & 1 & $16.7443(0.0896)$ & $18.9535(0.0549)$ & $23.3721(0.0694)$ & $25.5814(0.1015)$ & $26.6860(0.1211)$ & $27.3488(0.1336)$ \\
\hline & 5 & $16.7443(0.2987)$ & $18.9535(0.2072)$ & $23.3721(0.2735)$ & $25.5814(0.3989)$ & $26.6560(0.4665)$ & $27.3488(0.5062)$ \\
\hline \multirow[t]{2}{*}{40} & 1 & $16.7443(0.0896)$ & $20.6202(0.0604)$ & $28.3721(0.1570)$ & $32.2480(0.2371)$ & $34.1860(0.2793)$ & $35.3488(0.3048)$ \\
\hline & 5 & $16.7443(0.2987)$ & $20.6202(0.2208)$ & $28.3721(0.5416)$ & $32.2480(0.7293)$ & $34.1860(0.7991)$ & $35.3488(0.8342)$ \\
\hline \multirow[t]{2}{*}{50} & 1 & $16.7443(0.0896)$ & $22.2869(0.0733)$ & $33.3721(0.2613)$ & $38.9147(0.3806)$ & $41.6860(0.4379)$ & $43.3488(0.4709)$ \\
\hline & 5 & $16.7443(0.2987)$ & $22.2869(0.2586)$ & $33.3721(0.7560)$ & $38.9147(0.9029)$ & $41.6860(0.9415)$ & $43.3488(0.9573)$ \\
\hline
\end{tabular}

shrinkage estimates have a smaller risk for $\gamma=1$ than $\gamma=5$.

The parameters $a$ and $b$ could be estimated using a form of inference, called fiducial inference, see Casella and Berger (2001). The fiducial distribution of the parameter $\theta$ given the observations $\mathbf{r}=\left(r_{1}, \cdots, r_{m}\right)$ is used to describe our uncertainty about the parameter after $\mathbf{r}$ is observed when there was no a prior information about $\theta$. In this inference, $M(\mathbf{r}) L(\theta \mid \mathbf{r})$ is interpreted as a PDF for $\theta$, where $M(\mathbf{r})=\left(\int_{-\infty}^{\infty} L(\theta \mid \mathbf{r}) d \theta\right)^{-1}$. In our situation, $M(\mathbf{r})$ is given by

$$
M(\mathbf{r})=\frac{\left(\prod_{i=1}^{m} r_{i}\right) 2^{m-1} \Gamma(m-1)}{r_{m}^{2(m-1)}}, m>1 .
$$

Therefore, the fiducial distribution of $\theta$ given $\mathbf{r}$ is

$$
f i d(\theta)=\frac{r_{m}^{2(m-1)} e^{-\frac{r_{m}^{2}}{2 \theta}}}{2^{m-1} \Gamma(m-1) \theta^{m}}, \quad m>1,
$$

which is an inverse gamma, IGamma $\left(r_{m}^{2}, m-1\right)$. Then, the empirical estimates of $a$ and $b$ are $\hat{a}=r_{m}^{2}=2 m \hat{\theta}$ and $\hat{b}=m-1$. Therefore the empirical Bayesian estimators of $\theta$ is

$$
\hat{\theta}_{E B}(\mathbf{R})=\frac{2 m \hat{\theta}+\hat{a}}{2(m+\hat{b})}=\frac{2 m \hat{\theta}}{2 m-1} .
$$

Using the empirical estimates of $a$ and $b$, we have

$$
\hat{\lambda}^{\star}=\frac{m+(m-1) k}{2 m-1}, \quad \hat{\theta}_{0}=\frac{m \hat{\theta}}{m-1} .
$$


Therefore, the empirical generalized Bayesian shrinkage estimator of $\theta$ is

$$
\begin{aligned}
\hat{\theta}_{E G B S}^{(k)}(\mathbf{R}) & =\hat{\lambda}^{\star} \hat{\theta}+\left(1-\hat{\lambda}^{\star}\right) \hat{\theta}_{0} \\
& =\frac{m+(m-1) k}{2 m-1} \hat{\theta}+\frac{(m-1)(1-k)}{2 m-1} \frac{m \hat{\theta}}{m-1} \\
& =\frac{(2 m-k) \hat{\theta}}{2 m-1} .
\end{aligned}
$$

It is to be noted that the empirical generalized Bayesian shrinkage estimator and empirical Bayesian estimator are the same and the value of $\theta_{0}$ dose not affect the estimator.

Using a fiducial inference for estimation of $a$ and $b$, we have $\hat{a}=r_{5}^{2}=(12.94)^{2}=$ 167.44 and $\hat{b}=m-1=4$. Therefore, $\hat{\lambda}=m /(m+\hat{b})=0.56$ and the empirical estimate of $\theta_{0}$ is $\hat{\theta}_{0}=18.58$. Then, the Bayesian shrinkage estimate of $\theta$ is

$$
\hat{\theta}_{E G B S}^{(0)}=0.56(16.74)+(1-0.56)(20.93)=18.58 .
$$

\section{Concluding Remarks}

In this paper, we have obtained a generalized Bayesian shrinkage estimator for the scale parameter of a Rayleigh distribution on the basis of record samples under the RGL function in the presence of prior point information. The Bayesian and Bayesian shrinkage estimators are derived under the RGL and their risks are computed. Comparisons are made between these estimators via the relative efficiency using graphical and numerical methods. Our findings show that the Bayesian shrinkage estimator outperforms the MLE if the prior point information about the value of the parameter is not too far from its true value. Moreover, the Bayesian shrinkage estimator performs well with respect to the MLE for $\theta_{0}$ in the vicinity of $\theta$. In this case, the Bayesian shrinkage estimators with larger values of $b$ outperforms other estimators in the neighborhood of $\theta_{0}$, when $m$ and $\gamma$ held fixed. In practice, as the true value of the parameter is unknown, obtaining a point guess value is difficult. An empirical Bayesian procedure has been followed to obtain an estimated guess value of the parameter and utilizing so, the empirical Bayesian shrinkage estimates have been calculated. 


\section{Acknowledgements}

The authors are grateful to the referees for making helpful comments and suggestions.

\section{References}

Arnold, B. C., Balakrishnan, N., and Nagaraja, H. N. (1998), Records, John Wiley \& Sons, New York.

Baklizi, A., Asgharzadeh, A., and Sharifi, M. (2016), Estimation procedures based on preliminary test in exponential distribution under progressive censoring. Journal of Applied Statistical Science, 22, 141-152.

Belaghi, A. R., Arashi, M., and Tabatabaey, S. M. M. (2014), Improved confidence intervals for the scale parameter of Burr XII model based on record values. Computational Statistics, 29(5), 1153-1173.

Belaghi, A. R., Arashi, M., and Tabatabaey, S. M. M. (2015 a), On the construction of preliminary test estimator based on record values for the Burr XII model. Communications in Statistics-Theory and Methods, 44, 1-23.

Belaghi, A. R., Arashi, M., and Tabatabaey, S. M. M. (2015 b), Improved estimators of the distribution function based on lower record values. Statistical papers, 56(2), 453-477.

Casella, G. and Berger, R. (2001), Statistical Inference. 2nd Ed., Brooks/Cole.

Dey, S. and Das, M. K. (2007), A note on prediction interval for a Rayleigh distribution: Bayesian approach, Americam Journal of Mathematical and management Sciences, 27(12), $43-48$.

Dey, S. (2009), Comparison of Bayes estimators of the parameter and reliability function for Rayleigh distribution under different loss functions, Malaysian Journal of Mathematical Sciences, 3, 247-264.

Dey, S. and Dey, T. (2014), Statistical Inference for the Rayleigh distribution under progressively Type-II censoring with binomial removal. Applied Mathematical Modelling, 38, 974-982. 
Dey, S., Dey, T., and Maiti, S. (2015), Bayes shrinkage estimation of the parameter of Rayleigh distribution for progressive type-II censored data. Austrian Journal of Statistics, 44, 3-15.

Dey, S., Salehi, M., and Ahmadi, J. (2017), Rayleigh distribution revisited via ranked set sampling. Metron, 75(1), 69-85.

Kiapour, A. (2017), Bayes shrinkage estimator for the scale parameter of exponential distribution based on censored data. Journal of Statistical Sciences, 11, 119-131.

Kiapour, A. and Naghizadeh Qomi, M. (2016), Shrinkage preliminary test estimation under a precautionary loss function with applications on records and censored data, Journal of the Iranian Statistical Society, 15(2), 73-85.

Lawless, J. F. (2003), Statistical models and methods for lifetime data, Wiley, New York.

Meghnatisi, M. and Nematollahi, N. (2009), Inadmissibility of usual and mixed estimators of two ordered gamma scale parameters under reflected gamma loss function, Journal of Mathematical Extension, 3(2), 89-99.

Naghizadeh Qomi, M. (2017 a), Improved estimation in Rayleigh type-II censored data under a bounded loss utilizing a point guess value. Journal of the Iranian Statistical Society, 16, 51-66.

Naghizadeh Qomi, M. (2017b), Bayesian shrinkage estimation based on Rayleigh typeII censored data, Communications in Statistics-Theory and Methods, 46, 9859-9868.

Naghizadeh Qomi, M. and Barmoodeh, L. (2015), Shrinkage testimation in exponential distribution based on records under asymmetric squared log error loss. Journal of Statistical Research of Iran. 12, 225-238.

Naghizadeh Qomi, M., Nematollahi, N., and Parsian, A. (2015), On admissibility and inadmissibility of estimators after selection under reflected gamma loss function, Hacettepe Journal of Mathematics and Statistics, 44(5), 1109-1124.

Prakash, G. and Singh, D. C. (2008), Shrinkage estimation in exponential type-II censored data under LINEX loss, Journal of the Korean Statistical Society, 37, 53-61.

Prakash, G. and Singh, D. C. (2009), A Bayesian shrinkage approach in Weibull typeII censored data using prior point information. REVSTAT-Statistical Journal, 7(2), 171-187. 
Safariyan, A., Arashi, M., and Belaghi, A. R. (2018), Improved point and interval estimation of the stress-strength reliability based on ranked set sampling. Statistics, in press.

Thompson, J. R. (1968), Some shrunken techniques for estimating the Mean. Journal of the American Statistician Association, 63, 113-122.

Towhidi, M. and Behboodian, J. (1999), Estimation of a scale parameter under a reflected gamma loss functions, Iranian Journal of Science, 10, 256-269.

Towhidi, M. and Behboodian, J. (2002), Minimax estimation of a bounded parameter under some bounded loss functions, Far East Journal of Theoretical Statistics, 6(1), $39-48$.

Volterman, W., Belaghi, A. R., and Balakrishnan, N. (2018), Joint records from two exponential populations and associated inference, Computational Statistics, 33(1), 549562. 